\title{
On the Ionization Energy of the Outer Electrons of Atoms and Their Ions
}

\author{
Nikolay D. Gudkov ${ }^{1}$, Vladimir A. Shuvalov ${ }^{1,2}$ \\ ${ }^{1}$ Institute of Basic Biological Problems, Russian Academy of Sciences, Pushchino, Russia \\ ${ }^{2}$ A.N. Belozersky Institute of Physico-Chemical Biology, M.V. Lomonosov Moscow State University, \\ Moscow, Russia \\ Email: gudkov@issp.serpukhov.su,shuvalov@genebee.msu.ru
}

Received September 6, 2013; revised October 5, 2013; accepted October 27, 2013

Copyright (c) 2013 Nikolay D. Gudkov, Vladimir A. Shuvalov. This is an open access article distributed under the Creative Commons Attribution License, which permits unrestricted use, distribution, and reproduction in any medium, provided the original work is properly cited.

\begin{abstract}
An empirical formula is proposed to calculate the total binding energy of the outer electrons of atoms and their ions to a high accuracy. It is the authors' opinion that the validity of the proposed formula testifies that electronic shells have some "spatial structures", and the nature of which depends neither on the nucleus charge, nor on the number of the electrons in a shell.
\end{abstract}

Keywords: Complex Atoms; Electronic Shells; Ionization Energy

\section{Introduction}

It is generally known that “ $\cdots$ in a quantitative respect quantum mechanics is developed very poorly; $\cdots$ as a rigorous quantitative theory that it is not more than a theory of the hydrogen and helium atoms and of some other elementary systems" [1]. The well developed approximation methods allow us to calculate the energies of the stationary states of complex atoms with certain accuracy, however, numerical calculations turn out to be in this case extremely bulky and labor-consuming [2-5]. The more interesting is the result of [6], the author of which, using minimal computation means has estimated the binding energies of the outer electrons of more than 200 atoms and their ions to a high accuracy ( 1\% and higher). In his theoretical constructions, the author [6] utilizes, however, the ideas of the "old quantum theory" (with its, in particular, circular orbits of the electrons in atoms), and, for the understandable reasons, these speculations can not avoid apparent objections. In this paper, an attempt is made to present a more convincing version of the true background of the performed in [6] calculations.

\section{Results}

To a certain approximation, the total ionization (binding) energy $E_{n}$ of the electrons, which are in the outer shell of any atom (or ion), is determined by the number $N$ of the outer electrons, main quantum number $n$ of the shell to be considered and the nucleus charge $Z e$ :

$$
E_{n}=E_{n}(N, Z) \text {. }
$$

Whatever, the dependence (1) is, we always may, obviously, write it as

$$
E_{n}=N R y\left(\frac{Z_{n}}{n}\right)^{2}
$$

having equated thereby (quite formally) the mean binding energy per electron, $E_{n} / N$, to the ionization energy of a hydrogen-like atom [7], the nucleus charge of which, $Z_{n}{ }^{1}$, will be then some function of the same variables as $E_{n}$, namely, as it follows from Equation (2):

$$
Z_{n}=Z_{n}(N, Z)=n\left(\frac{E_{n}}{N R y}\right)^{1 / 2} .
$$

In Figures 1-3 are shown the results of $Z_{n}$ calculations with this formula based on experimental data for ionization potentials of the atoms and their cations [8] with different values both of nucleus charge and main quantum number of the outer electron shell (for more details see Figure captions).

\footnotetext{
${ }^{1}$ Appearing in Equation (2) the unit of energy ("rydberg") $R y=$ $13.6057 \mathrm{ev}$ [7], and here and below by "nucleus charge" is taken its value in units of $e$.
} 


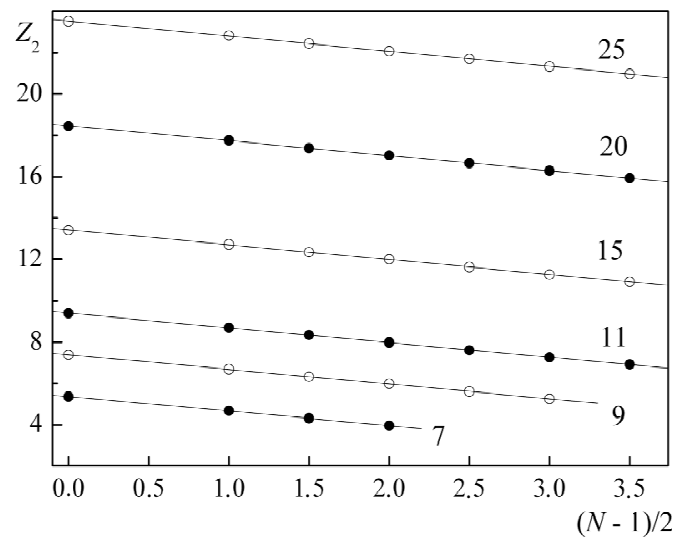

Figure 1. Dependence of the quantity $Z_{n}$, calculated from the formula (3) using experimental values $E_{n}$ from [8], on the number $N$ of electrons in the outer shell of atoms and their cations for the shells with the main quantum number $n=2$. The numbers of the curves give the position of the corresponding elements in the periodic table (i.e., coincide with the values of $Z$ ), and, hence, the presented data refer to: $\mathrm{N}-\mathrm{N}^{4+}$ (i.e., from $\mathrm{N}$ to $\mathrm{N}^{4+}$ ) (7), $\mathrm{F}-\mathrm{F}^{6+}(9), \mathrm{Na}^{+}-\mathrm{Na}^{8+}(11), \mathrm{P}^{5+}-$ $\mathrm{P}^{12+}(15), \mathrm{Ca}^{10+}-\mathrm{Ca}^{17+}(20), \mathrm{Mn}^{15+}-\mathrm{Mn}^{22+}(25)$. In order not to overload the figure, the results of calculations $Z_{2}$ for the shells: $\mathrm{C}_{-} \mathrm{C}^{3+}, \mathrm{O}-\mathrm{O}^{5+}, \mathrm{Ne}-\mathrm{Ne}^{7+}, \mathrm{Mg}^{2+}-\mathrm{Mg}^{9+}, \mathrm{Al}^{3+}-\mathrm{Al}^{10+}, \mathrm{Si}^{5+}-$ $\mathrm{Si}^{12+}, \mathrm{S}^{6+}-\mathrm{S}^{13+}, \mathrm{Cl}^{7+}-\mathrm{Cl}^{14+}, \mathrm{Ar}^{8+}-\mathrm{Ar}^{15+}, \mathrm{K}^{9+}-\mathrm{K}^{16+}, \mathrm{Sc}^{11+}-\mathrm{Sc}^{18+}$, $\mathrm{Ti}^{12+}-\mathrm{Ti}^{19+}, \mathrm{V}^{13+}-\mathrm{V}^{20+}, \mathrm{Cr}^{14+}-\mathrm{Cr}^{21+}, \mathrm{Fe}^{16+}-\mathrm{Fe}^{23+}, \mathrm{Co}^{17+}-\mathrm{Co}^{24+}$, $\mathrm{Ni}^{18+}-\mathrm{Ni}^{25+}, \mathrm{Cu}^{19+}-\mathrm{Cu}^{26+}$ are not shown.

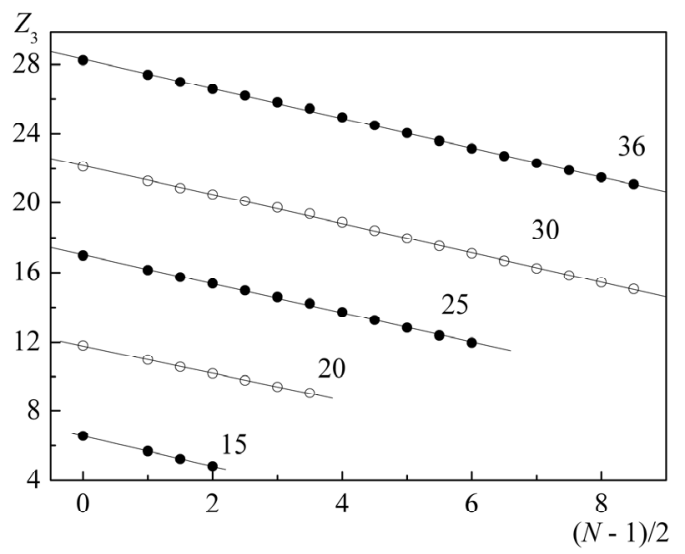

Figure 2. The same as in Figure 1, but for the shells of $n=3$ : $\mathrm{P}^{\mathrm{P}^{4+}}(15), \mathrm{Ca}^{2+}-\mathrm{Ca}^{9+}(20), \mathrm{Mn}^{2+}-\mathrm{Mn}^{14+}(25), \mathrm{Zn}^{2+}-\mathrm{Zn}^{19+}$ (30), $\mathrm{Kr}^{8+}-\mathrm{Kr}^{25+}$ (36). As before, the results are not shown for the shells: $\mathrm{Si}_{-\mathrm{Si}^{3+}}, \mathrm{S}_{-} \mathrm{S}^{5+}$, Cl-Cl ${ }^{6+}, \mathrm{Ar}-\mathrm{Ar}^{7+}, \mathrm{K}^{+}-\mathrm{K}^{8+}, \mathrm{Sc}^{2+}-\mathrm{Sc}^{10+}$, $\mathrm{Ti}^{2+}-\mathrm{Ti}^{11+}, \mathrm{V}^{2+}-\mathrm{V}^{12+}, \mathrm{Cr}^{+}-\mathrm{Cr}^{12+}, \mathrm{Fe}^{2+}-\mathrm{Fe}^{15+}, \mathrm{Co}^{2+}-\mathrm{Co}^{14+}, \mathrm{Ni}^{2+}-$ $\mathrm{Ni}^{17+}, \mathrm{Cu}^{+}-\mathrm{Cu}^{18+}$.

From the data presented (i.e., from experimental data for the energies $E_{n}$ ) it of necessity follows that

$$
Z_{n}=Z_{n}^{\prime}-k_{n} \frac{N-1}{2},
$$

where, as seen from the Figures, parameter $Z_{n}^{\prime}$ is about $Z$, whereas the slope $k_{n} \approx$ const $(Z)$ but with regularity (monotonically increasing) depends on $n$ :

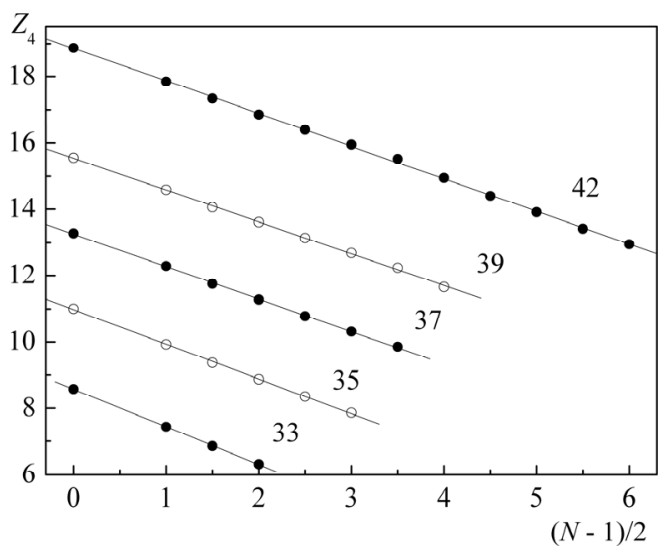

Figure 3. The same as in the previous figures for the case of $n=4: \mathrm{As}^{-A s^{4+}}$ (33), Br-Br${ }^{6+}(35), \mathrm{Rb}^{+}-\mathrm{Rb}^{8+}(37), \mathrm{Y}^{2+}-\mathrm{Y}^{10+}$ (39), $\mathrm{Mo}^{+}-\mathrm{Mo}^{13+}(42)$. For the same considerations as before (Figures 1 and 2), the data for: $\mathrm{Ge}-\mathrm{Ge}^{3+}, \mathrm{Se}^{-\mathrm{Se}^{5+}}, \mathrm{Kr}-\mathrm{Kr}^{7+}$, $\mathrm{Sr}^{2+}-\mathrm{Sr}^{9+}$ are not given. Points calculated in the case $N=2$ are not shown in the figures: in all cases these points lie outside the straight lines drawn through the points $\left(Z_{n},(N-1) / 2\right)$ with $N \neq 2$ (see Figure 4 as illustration). This property of the closed $s$-shells is left without comments, and, consequently, the analysis and interpretation of the presented data do not extend on these shells.

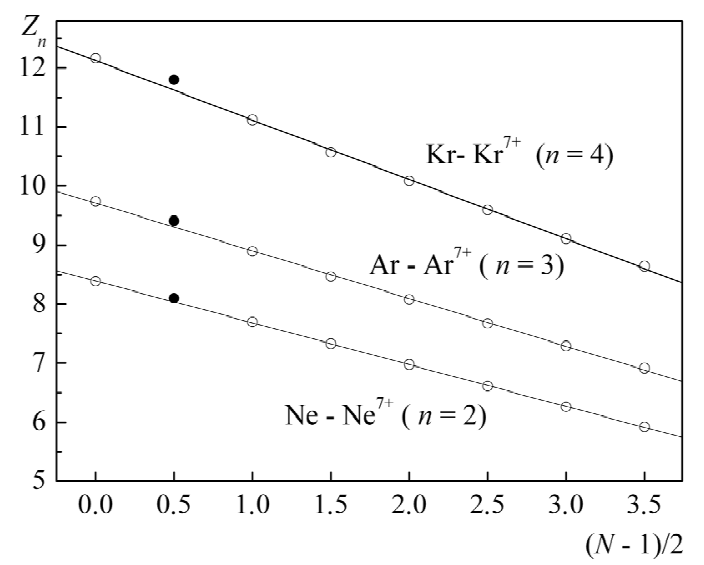

Figure 4. Explanations in the text (see the third sentence in the caption to Figure 3).

$$
k_{n}=\left\{\begin{array}{l}
0.72 \pm 0.01, n=2 \\
0.83 \pm 0.01, n=3, \\
1.04 \pm 0.07, n=4
\end{array}\right.
$$

Thus, the experimental data for the energies of ionization of atoms and their cations testify the existence of one more-along with the main quantum number $n$ "invariant of electronic shell": at fixed $n$ the value of dimensionless parameter $k_{n}$ does not depend either on the nucleus charge, or on the number of electrons in the shell. This interesting observation explains, in particular, the mentioned above results of the author [6] on estimation of the value $E_{n}$ for a large number of atoms and their 
ions: resulting from Equations (2) and (4) is an empirical formula ${ }^{2}$

$$
E_{n}=N R y\left[\frac{Z_{n}^{\prime}-k_{n}(N-1) / 2}{n}\right]^{2}
$$

coinciding (within notations and a layout) with equality (18) from the work [6], where this formula was, in fact, postulated by the author ${ }^{3}$.

\section{Discussion}

To understand (even at first approximation) the physical meaning and importance of the found relation (4) and (5), or, which is the same, of the formula (6) which follows from Equations (2), (4), let us proceed as follows. Without taking into account spin effects, the Hamiltonian of the considered set of electrons is the sum

$$
H=T+U+U^{\prime},
$$

where $T$ is an operator of kinetic energy, $U$ - energy of Coulomb interaction of the outer electrons with the nucleus and the electrons of the inner shells, $U^{\prime}$ is electrostatic energy of mutual repulsion of the outer layer electrons, and, thus,

$$
H=T-\sum_{i=1}^{N} e^{2}\left(\frac{Z}{r_{i}}-\sum_{j=1}^{N_{\text {in }}} \frac{1}{\rho_{i j}}\right)+\sum_{k \neq i=1}^{N} \frac{e^{2}}{d_{i k}} .
$$

Here $r_{i}$-the distance of the $i^{\text {th }}$ electron to the nucleus, $\rho_{i j}$-the distance between the outer electron with the number $i$ and the $j^{\text {th }}$ electron of an inner layers, $N_{\text {in }}$ - the number of electrons in these layers, $d_{i k}$-the mutual distance of two electrons of the outer shell.

If we now denote by $|n\rangle$ a wave function (a vector) of the lowest state of the system under consideration, so that

$$
H|n\rangle=E_{n}|n\rangle,
$$

then ionization energy will be

$$
E_{n}=\langle n|H| n\rangle /\langle n \mid n\rangle .
$$

Substitution of Equation (7) into Equation (8) gives

$$
E_{n}=\langle T\rangle-\sum_{i} e^{2}\left(Z\left\langle\frac{1}{r_{i}}\right\rangle-\sum_{j}\left\langle\frac{1}{\rho_{i j}}\right\rangle\right)+\sum_{k \neq i} e^{2}\left\langle\frac{1}{d_{i k}}\right\rangle,
$$

where angle brackets stand for the mean values of the

${ }^{2}$ The error of the $E_{n}$ values calculated with this formula (with the $k_{n}$ value taken from equalities (5) and with the charge $Z_{n}^{\prime}$ determined from the data presented in Figures 1-3) lies between a few percent down to hundredths of a percent, and, on the average, the accuracy of the formula may be estimated by $1 \%$.

${ }^{3}$ In any case, an attempt of the author [6] to obtain formula (6) as a formal consequence of "old quantum theory", i.e., "to derive" the formula, using the concept of circular Bohr orbits, is not possible to recognize as successful for a variety of reasons. corresponding quantities.

Taking into account that the number of terms in the sum with $k \neq i$ equals to $N(N-1) / 2$, let us rewrite the right-hand side of Equation (9) in the form

$$
E_{n}=N\left[\bar{T}-\frac{e^{2}}{r_{n}}\left(Z-\frac{r_{n}}{\rho_{n}} N_{\text {in }}-\frac{r_{n}}{d_{n}} \frac{N-1}{2}\right)\right] .
$$

Here $\bar{T}(=\langle T\rangle / N)$ is the mean kinetic energy of an outer electron, and, $r_{n}, \rho_{n}$ and $d_{n}$ are given by:

$$
\begin{gathered}
\frac{N}{r_{n}}=\sum_{i}\left\langle\frac{1}{r_{i}}\right\rangle, \frac{N N_{\text {in }}}{\rho_{n}}=\sum_{i, j}\left\langle\frac{1}{\rho_{i j}}\right\rangle, \\
\frac{N(N-1)}{2 d_{n}}=\sum_{k \neq i}\left\langle\frac{1}{d_{i k}}\right\rangle .
\end{gathered}
$$

For the mean binding energy per electron we obtain from Equation (10):

$$
E_{n} / N=\bar{T}-\frac{\hat{Z} e^{2}}{r_{n}}
$$

where the following notation is used:

$$
\hat{Z}=Z-\frac{r_{n}}{\rho_{n}} N_{\text {in }}-\frac{r_{n}}{d_{n}}(N-1) / 2 .
$$

An expression in the right side of Equation (11) represents the energy of an electron which moves in the filed of the nucleus with the charge $\hat{Z}$ and occupies, according to initial assumption, an orbital of the main quantum number $n$. Comparing Equations (11) and (2), we conclude that it is possible then to set:

$$
Z_{n}=\hat{Z} .
$$

which means, in its turn, that appearing in Equation (4) empirical parameters ought to be assigned, when compared to Equation (12), the following meanings:

$$
\begin{gathered}
Z_{n}^{\prime}=Z-\kappa_{n} N_{\text {in }}, \\
k_{n}=r_{n} / d_{n} .
\end{gathered}
$$

where the notation $\kappa_{n}=r_{n} / \rho_{n}$ was introduced.

Equality (14) permits a simple physical interpretation, namely: $Z_{n}^{\prime}$ is the "effective" charge of the nucleus screened by the electrons of the inner shells, and the factor $\kappa_{n}$ has, respectively, the meaning of the coefficient of the screening, i.e., of the effect, caused by interaction of the electrons of the outer shell with the electrons of the inner layers. Taking into account relations (12)-(15), similar reasoning is true in case of Equation (4) too: mutual repulsion of the outer shell electrons also amounts to the screening of positive nucleus charge, and the parameter $k_{n}$ is, thereby, a measure of the efficiency ("a coefficient”) of such ("outer") screening.

The discovered coefficient $k_{n}$ "invariance", i.e., -in accordance with Equations (5), (15)—the ratio $r_{n} / d_{n}$ 
independence of nucleus charge and the number of the electrons in the shells having the same quantum number $n$, may mean, for example, that electronic shells (layers) are "spatially structured": the electrons experience something like random "migration" between nodes of a certain spatial lattice (with the number of vertexes $2 n^{2}$ ), inscribed into a sphere of radius $r_{n}$. For example, for $n=2$ the "structure" of the electronic shell may be close to a cube: the cubic lattice, being centrosymmetrical, has the required number of nodes, and, as easy to calculate, the ratio of the mean inverse distance between the vertexes to the inverse radius of a circumscribed sphere (i.e., the ratio $r_{2} / d_{2}$ for this case) equals to 0.705 (cf. Equation (5) for $n=2)^{4}$.

It is more difficult to imagine the "portraits" of shells with $n=3,4$. It is easy to construct centrosymmetrical lattice with sufficient number of the vertexes and appropriate mean inverse distance between them-the problem is to choose from a multitude of such lattices those which could correspond to physical reality. The problem of "selection rules" requires, apparently, a more thorough theoretical analysis.

\section{Conclusion}

In conclusion, it should be emphasized that the purpose of this communication is to draw attention to validity of a non trivial, as it seems to us, empirical dependence (6) of total ionization energy of the outer electrons of atoms and their cations on the number of electrons in the outer shell, the shell main quantum number and charge of the nucleus of an atom or an ion. As for proposed interpreta- tion of the regularity presented above, the authors, of course, do not consider it as final being quite aware of the vulnerability of their constructions and of their qualitative, generally speaking, nature.

\section{REFERENCES}

[1] V. V. Tolmachev, "Inroduction of the Translation Editor," In: H. Lipkin, Quantum Mechanics (Russian), MIR, Moscow, 1977, pp. 5-8.

[2] A. S. Davydov, “Quantum Mechanics,” Pergamon Press, Oxford, 1985.

[3] A. Messiah, “Quantum Mechanics,” Dover Publications, Mineola, 1999.

[4] G. L. Malli, A. B. F. Da Silva and Y. Ishikawa, Physical Review, Vol. A47, 1993, pp. 143-146.

[5] S. N. Datta, International Journal of Quantum Chemistry, Vol. 56, 1995, pp. 91-95. http://dx.doi.org/10.1002/qua.560560204

[6] V. A. Shuvalov, Biochimiya (Russian), Vol. 68, 2003, pp. 1333-1354.

[7] L. D. Landau and E. M. Lifshitz, "Quantum Mechanics: Non-Relativistic Theory,” Pergamon Press, Oxford, 1977.

[8] D. R. Lide, Ed., "CRC Handbook of Chemistry and Physics,” 88th Edition, CRC Press, Boca Raton, 2007-2008, pp. 10-203-10-205.

[9] G. N. Lewis, Journal of the American Chemical Society, Vol. 38, 1916, pp. 762-785. http://dx.doi.org/10.1021/ja02261a002

[10] I. Langmuir, Journal of the American Chemical Society, Vol. 41, 1919, pp. 868-934. http://dx.doi.org/10.1021/ja02227a002
${ }^{4}$ It may be noteworthy to remember here of an article by Gilbert Newton Lewis [9] widely known some time ago, concerning his "theory of cubic atom" (in the development of which was published no less well cited paper of Irving Langmuir [10] on "concentric theory of atomic structure"). 